\title{
Long Non-Coding RNA MEG3 Downregulation Triggers Human Pulmonary Artery Smooth Muscle Cell Proliferation and Migration via the p53 Signaling Pathway
}

\author{
Zengxian Sun ${ }^{a}$ Xiaowei Niec,d Shuyang Sun ${ }^{\text {e }}$ Shumin Dong ${ }^{f}$ Chunluan Yuang
}

Yanli Li ${ }^{a}$ Bingxin Xiao ${ }^{a}$ Dong Jie ${ }^{a}$ Yun Liu,ab

aDepartment of Pharmacy, The First People's Hospital of Lianyungang, Lianyungang, bepartment of Clinical pharmacology laboratory, The Affiliated Lianyungang Hospital of Xuzhou Medical University/The First People's Hospital of Lianyungang, Lianyungang, 'Jiangsu Key Laboratory of Organ Transplantation, Wuxi People's Hospital Affiliated to Nanjing Medical University, Nanjing, dDepartment of Clinical Laboratory Science, Wuxi People's Hospital of Nanjing Medical University, Nanjing, 'Overseas Education College, Nanjing Tech University, Nanjing, fDepartment of thoracic surgery, The First People's Hospital of Lianyungang, Lianyungang, 9Department of Oncology, The First People's Hospital of Lianyungang, Lianyungang, China

\section{Key Words}

Long non-coding RNAs • Pulmonary arterial hypertension • Proliferation • p53 - Pulmonary artery smooth muscle cells

\begin{abstract}
Background/Aims: Increasing evidence has demonstrated a significant role of long noncoding RNAs (IncRNAs) in diverse biological processes, and many of which are likely to have functional roles in vascular remodeling. However, their functions in pulmonary arterial hypertension (PAH) remain largely unknown. Pulmonary vascular remodeling is an important pathological feature of $\mathrm{PAH}$, leading to increased vascular resistance and reduced compliance. Pulmonary artery smooth muscle cells (PASMCs) dysfunction is involved in vascular remodeling. Long noncoding RNAs are potential regulators of PASMCs function. Herein, we determined whether long noncoding RNA-maternally expressed gene 3 (MEG3) was involved in PAH-related vascular remodeling. Methods: The arterial wall thickness was examined by hematoxylin and eosin (H\&E) staining in distal pulmonary arteries (PAs) isolated from lungs of healthy volunteers and PAH patients. The expression level of MEG3 was analyzed by qPCR. The effects of MEG3 on human PASMCs were assessed by cell counting Kit- 8 assay, BrdU incorporation assay, flow cytometry, scratch-wound assay, immunofluorescence, and western blotting in human PASMCs. Results: We revealed that the expression of MEG3 was significantly downregulated in lung and PAs of patients with PAH. MEG3 knockdown affected PASMCs Z. Sun and X. Nie contributed equally to this work.


proliferation and migration in vitro. Moreover, inhibition of MEG3 regulated the cell cycle progression and made more smooth muscle cells from the $G_{0} / G_{1}$ phase to the $G_{2} / M+S$ phase and the process could stimulate the expression of PCNA, Cyclin A and Cyclin E. In addition, we found that the p53 pathway was involved in MEG3-induced smooth muscle cell proliferation. Conclusions: This study identified MEG3 as a critical regulator in PAH and demonstrated the potential of gene therapy and drug development for treating PAH.

(C) 2017 The Author(s)

Published by S. Karger AG, Basel

\section{Introduction}

Pulmonary arterial hypertension (PAH) is an intractable disease, a key feature of which is pulmonary vascular remodeling. Vascular remodeling is a central process in the PAH pathogenesis, which is associated with the accumulation of extracellular matrix and the proliferation and hypertrophy of vascular smooth muscle cells (VSMCs). These processes contribute to the medial hypertrophy and muscularization, neointima formation and plexiform lesions development, leading to the obliteration of precapillary pulmonary arteries and sustained elevation of pulmonary arterial pressure $[1,2]$. Although advancements have been made in elucidating the pathogenesis of $\mathrm{PAH}$, the underlying molecular mechanisms of PAH are still poorly understood.

LncRNAs, which are commonly defined as noncoding transcripts $>200$ nucleotides in length without evident protein coding function, were once considered to be transcriptional "noise". Accumulating studies have revealed that lncRNAs play significant roles in multiple biological processes, including cell proliferation [3] and differentiation [4], cell fate determination [5], and apoptosis [6]. Although most studies have been focused on the oncogenic or tumor suppressive functions of IncRNAs, some studies have also demonstrated that aberrant expression profiles of lncRNAs are associated with other human diseases, including cardiovascular disease [7,8]. However, it is not yet clear whether lncRNA is involved in the regulation of pulmonary vascular remodeling.

lncRNA gene, maternally expressed gene 3 (MEG3) is located on chromosome 14q32.3 in humans [9]. The loss of MEG3 expression has been observed in various types of cancers, including non-small cell lung cancer (NSCLC) [10], gastric cancer [11] and bladder cancer [12]. Ectopic expression of MEG3 inhibited cell proliferation and promoted cell apoptosis in various cancer cells $[13,14]$. These studies suggest the MEG3 may be a tumor suppressor. Moreover, its tumor suppressor activity was mediated by interaction with p53-dependent transcription [15]. Since PAH and cancers share some common features, like excessive cellular proliferation and apoptosis resistance $[16,17]$, we were interested in the question of whether MEG3 is involved in PAH pathogenesis. To this end, we studied the expression profile of MEG3 in PAH patients and further investigated the effects of MEG3 on the biological behaviors of pulmonary artery smooth muscle cells (PASMCs) and their underlying molecular mechanisms. We found that lncRNA MEG3 was low-expressed in PAH patients and human pulmonary artery smooth muscle cells (PASMCs) in hypoxia. MEG3 regulates PASMCs function in vitro. Our data emphasize that silencing of MEG3 induces proliferation and migration of human PASMCs. In addition, further study found that MEG3 changed the cell cycle associated proteins PCNA, Cyclin A and Cyclin E in human PASMCs after transfection of si-lncRNA MEG3. Results displayed that the protein expression of PCNA, Cyclin A and Cyclin E was elevated by MEG3 knockdown, and data was consistent in hypoxia condition. Moreover, we found that MEG3 could activate the p53 signaling pathway, and its intervention affects p53 nuclear translocation. Taken together, these results indicate that lncRNAs, especially MEG3 plays an important role in PAH development and may be a potential therapeutic target for patients with PAH. 


\section{Cellular Physiology Cell Physiol Biochem 2017;42:2569-2581 \begin{tabular}{l|l|l} 
and Biochemistry Published onlıne: August 23, 2017 & $\begin{array}{l}\text { (c) } 2017 \text { The Author(s). Published by S. Karger AG, Basel } \\
\text { www.karger.com/cpb }\end{array}$
\end{tabular}}

\section{Material and Methods}

\section{Materials}

CycleTEST $^{\text {тм }}$ PLUS DNA Reagent Kit was provided from BD Biosciences (Bedford, MA). Bromodeoxyuridine (BrdU) proliferation assay kit was purchased from Millipore Corporation (Billerica, MA). Antibodies against p53, Cyclin A, Cyclin E, MDM2 and ACTB were purchased from Santa Cruz Biotechnology Inc. (Santa Cruz, USA). Enhanced chemiluminesence (ECL) reagents were from Amersham International (Amersham, UK). All other reagents were from common commercial sources.

\section{Lung Tissue Preparation and H\&E staining}

Human lung samples of PAH were obtained from lung transplantation patients (PAH) or localized lung cancer patients (normal) for lobectomy or pneumonectomy in the Xuzhou Medical University Affiliated Lianyungang Hospital under the procedures approved by the Xuzhou Medical University Ethical Committee for Use of Human Samples.

The lung tissues were sliced into tissue blocks, and immersed in 4\% paraformaldehyde for overnight fixation. Then fixed tissues were dehydrated, cleared, and embedded in paraffin wax. The paraffin blocks were cut into $5 \mu \mathrm{m}$ thick sections and stained with hematoxylin and eosin (H\&E) according to our previously published protocol [18].

\section{Cell culture}

Human PASMCs were purchased from Gibco (Life Technologies, Zug, Switzerland) and maintained in SmGM-2 BulletKit media (Lonza, Basel, Switzerland) containing 5\% (volume/volume) heat-inactivated fetal bovine serum (FBS; Gibco, Carlsbad, CA), $0.5 \mathrm{ng} / \mathrm{ml}$ human recombinant epidermal growth factor, $2 \mathrm{ng} / \mathrm{ml}$ human recombinant fibroblast growth factor, $5 \mu \mathrm{g} / \mathrm{ml}$ insulin, $50 \mu \mathrm{g} / \mathrm{ml}$ gentamicin, $10 \mathrm{U} / \mathrm{ml}$ penicillin and $10 \mu \mathrm{g} / \mathrm{ml}$ streptomycin. Cells were incubated at $37^{\circ} \mathrm{C}$ in a humidified $5 \% \mathrm{CO}_{2}$ incubator. For experiments in a hypoxic condition, human PASMCs were cultured with a gas mixture containing $92 \% \mathrm{~N}_{2}-5 \% \mathrm{CO}_{2}-3 \% \mathrm{O}_{2}$. Before each experiment, the human PASMCs were quiesced for $24 \mathrm{~h}$ in SmGM-2 BulletKit media without serum. Then cells were treated with different reagents as described in the results.

\section{Small interfering RNA and transfection}

The sequence of siRNA against IncRNA MEG3 and scrambled negative control siRNA (si-NC) were synthesized by GenePharma (Shanghai GenePharma Co, Shanghai, China). The target sequences of these siRNAs were follows: si-IncRNA MEG3, 5'-GGGCTTCTGGAATGAGCAT-3'; si-NC, 5'-UUCUCCGAAC GUGUCACGUTT-3'. Overexpression of p53 was performed according previously published protocol [19]. Human PASMCs cultured on six-well plate were transfected with the si- IncRNA MEG3 or si-NC using Lipofectamine2000 (Invitrogen, Shanghai, China) according to the manufacturer's instructions. 24 h post transfection, cells were harvested for qRT-PCR and Western blotting analyses.

\section{Quantitative real-time PCR}

Total RNAs were extracted from lung tissues, pulmonary arteries (PAs) or human PASMCs using the Trizol reagent according to the manufacturer's instructions and the quality and concentrations of total RNA were detected by ultraviolet spectrophotometry. Reverse transcription (RT) reaction was performed using the PrimeScript RT reagent kit (Qiagen). Real-time PCR was performed in triplicate on 7500 Fast RealTime PCR System (Applied Biosystems) using SYBR Premix DimerEraser kit following the manufacturer's instructions. GAPDH was used as a housekeeper gene for the qRT-PCR reactions. GAPDH was used as housekeeper gene for qRT-PCR reactions. The $2^{-\Delta \Delta \mathrm{Ct}}$ method was used to calculate the expression of lncRNA MEG3 in tissue and cell samples [20]. The primer sequences for PCR are listed in Table 1.

\section{Western blotting}

Human PASMCs were placed in the smooth muscle cell medium without serum for $24 \mathrm{~h}$ when reached $60 \%$ confluence. Cells were collected after $24 \mathrm{~h}$ of transfection with si-NC or si-lncRNA MEG3 treatment and resuspended in RIPA lysis buffer (Santa Cruz) for $30 \mathrm{~min}$ on ice. After $30 \mathrm{~min}$ of lysis, cell lysates were sonicated for $1 \mathrm{~min}$ and centrifuged at $14,000 \mathrm{~g}$ for $15 \mathrm{~min}$, and the concentrations of the proteins were measured by BCA kit (Dingguo Company). Samples containing 20 ug total protein from each sample were 


\section{Cellular Physiology Cell Physiol Biochem 2017;42:2569-2581 \begin{tabular}{l|l|l} 
and Biochemistry Published onlIne: August 23, 2017 & $\begin{array}{l}\text { C) } 2017 \text { The Author(s). Published by S. Karger AG, Basel } \\
\text { www.karger.com/cpb }\end{array}$ \\
\hline
\end{tabular}}

Sun et al.: Role of MEG3 in Pulmonary Arterial Hypertension

Table 1. Primer sequences used in real-time PCR

\begin{tabular}{ll}
\hline Oligonucleotides & Sequence \\
\hline MEG3 forward & 5'-CTGCCATCTACACCTCACG-3' \\
MEG3 reverse & 5'-CTCTCCGCCGTCTGCGCTAGGGGCT-3' \\
GAPDH forward & 5'-GAAGGTGAAGGTCGGAGTC-3' \\
GAPDH reverse & 5'-GAAGATGGTGATGGGATTTC-3' \\
\hline
\end{tabular}

used for SDS-PAGE (Bio-Rad Laboratories), and Western blotting was performed according to the protocol as described previously [18].

\section{Immunofluorescence}

For immunofluorescence staining, the human PASMCs were fixed with $4 \%$ paraformaldehyde (PFA) for $30 \mathrm{~min}$, washed three times with cold PBS and permeabilized in 0.4\% triton-X 100 (Sigma-Aldrich) for $5 \mathrm{~min}$. After washing with PBS, the cells were blocked in $1 \%$ BSA for $30 \mathrm{~min}$ and incubated overnight at $4^{\circ} \mathrm{C}$ with p53 antibody with 1:200 dilution in 1\% BSA. After extensive washes with PBS for $30 \mathrm{~min}$, the cells were incubated with the secondary antibody IgG (Sigma) conjugated with rhodamine at a 1:200 dilution for 1 hour at room temperature. DAPI (4, 6-diamidino-2-phenylindole) was used to label the nuclei, and the cells were observed for fluorescence images under a fluorescence microscope (Nikon).

\section{Scratch-Wound assay}

The confluent human PASMCs cultured in 6-well plates were wounded by pipette tips, creating an acellular 1-mm-wide lane per well, and the detached cells were washed out by PBS [21]. Then the cells were transfected with si-NC or si-IncRNA MEG3 under both normal and hypoxic conditions. Wounded areas were photographed at zero time. After $24 \mathrm{~h}$ of incubation, photographs were taken from the same areas as those recorded at zero time.

\section{Proliferation Assays}

Cell Counting Kit-8 (CCK-8) assay. Human PASMCs were cultured in 96-well microtitration plates and then the cells were subjected to growth arrest by serum starvation for $24 \mathrm{~h}$. The cells were transfected with si-NC, si-IncRNA MEG3, or si-IncRNA MEG3+si-p53. After $24 \mathrm{~h}$ of the incubation at $37^{\circ} \mathrm{C}$, the proliferation potential of human PASMCs was determined by the CCK-8 assay (Dojin Laboratories, catalog no: CK04) according to the manufacturer's instruction.

DNA BrdU incorporation assay. Cell proliferation was assessed by 5-bromodeoxyuridine (BrdU) incorporation. Human PASMCs were seeded into 96-well cell culture plates at a density of $1 \times 10^{4}$ cells /well. When the cells were treated with MEG3 knockdown or hypoxia, BrdU was incorporated into proliferating cells and was added at 2-24 h prior to the end of the test reagent incubation. Following incorporation, cells were fixed by the fixing solution at room temperature for $30 \mathrm{~min}$. The ensuing steps were carried out according to the manufacturer's protocol. Finally, the optical absorbance was measured by a spectrophotometer microplate reader at dual wavelengths of $450 / 550 \mathrm{~nm}$.

\section{Cell cycle analysis}

To study the cell cycle induced by MEG3 knockdown or hypoxia, we analyzed the proportion of cells in $G_{0} / G_{1}, S$ and $G_{2} / M$ phases by flow cytometry, as reported recently [16]. After the cells were subjected to si-IncRNA MEG3 or hypoxia, they were harvested by trypsinization, then centrifuged and suspended with $1 \mathrm{ml}$ of cold PBS. Following twice washes with PBS, the cells were resuspended and fixed by $70 \%$ cold ethanol and were kept at $4^{\circ} \mathrm{C}$. Before detection, the fixed cells were centrifuged at $300 \times \mathrm{g}$ for $5 \mathrm{~min}$, and the supernatant fluid was discarded. The cell sediment was resuspended in $200 \mathrm{ul} \mathrm{PBS}$, and $200 \mathrm{ul}$ RNase A was added. The suspension was then incubated at room temperature for $10 \mathrm{~min}$. Then $200 \mathrm{ul}$ of propidium iodide was added and mixed, and the reaction was carried out at $4^{\circ} \mathrm{C}$ in dark for $10 \mathrm{~min}$. Finally, the cells were filtered once through 400-mesh sieves berore undergoing by flow cytometer. 
Statistical analysis

Experimental data are presented as mean \pm S.E.M. Statistical analysis was performed with one-way ANOVA (analysis of variance), followed by Dunnett's test where appropriate. Differences were considered statistical significant at $\mathrm{P}<0.05$.

\section{Results}

MEG3 expression is down-regulated in both PAH tisues and hypoxic PASMCs

The morphology of pulmonary vessels was analyzed with H\&E staining to show potential correlations of the morphological changes with remodeling. Wall thickness of medium sized PAs from PAH patients was significantly increased compared with normal (Fig. 1A, B). To determine whether IncRNA MEG3 was differentially expressed in the lung tissues and PAs between PAH and normal tissues, a total of 5 paired PAH tissues and normal lung tissues and PAs were analyzed for MEG3 expression using qRT-PCR. As shown in Fig. 1C, D, the mean expression level of MEG3 in lung tissues and PAs from PAH patients was significantly lower than that of the normal tissues. The results suggested that the MEG3 expression in PAH patients was significantly downregulated compared to that of normal controls. Consistently, MEG3 expression was also downregulated in a time-dependent manner in hypoxic PASMCs (Fig. 1E).

\section{MEG3 knockdown promotes proliferation and migration of human PASMCs}

To determine the function of MEG3 in human PASMCs under hypoxia condition, we investigated the effects of MEG3 knockdown on the proliferation and migration of human PASMCs. We designed small interfering RNA (siRNA) to inhibit lncRNA MEG3 expression.

Fig. 1. qRT-PCR analysis of IncRNA MEG3 in lung tissues of PAH and PASMCs in hypoxic exposure. (A) H\&E staining of human lungs found that the wall thickness in $\mathrm{PAH}$ was higher compared with normal humans; (B) Histogram analysis showed that the wall thickness was significantly increased in PAH patients as compared to normal controls; (C, D) qRT-PCR analysis of MEG3 expression in lung and PAs tissues from patients with $\mathrm{PAH}$ and healthy lung donors (Normal); (E) qRT-PCR analysis of MEG3 expression levels in human PASMCs. $\mathrm{n}=5$, ${ }^{*} \mathrm{p}<$ $0.05,{ }^{* * *} \mathrm{p}<0.01$. Normal, healthy lung donors; $\mathrm{PAH}$, pulmonary arterial hypertension patients; PASMCs, pulmonary artery smooth muscle cells.

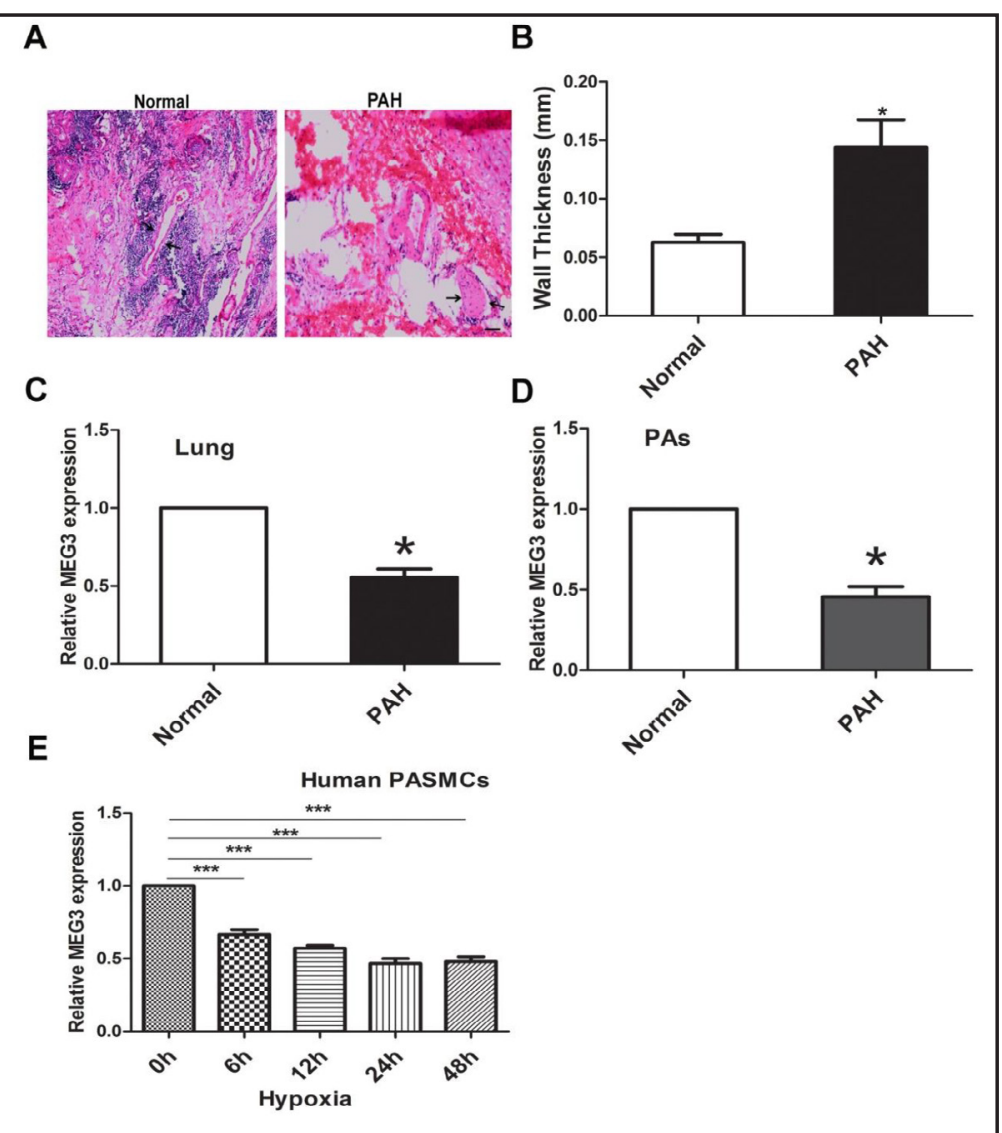


Fig. 2. MEG3 knockdown promotes human PASMCs proliferation in vitro. (A, B) MEG3 expression level in human PASMCs following the treatment with si-NC or si-lncRNA MEG3; (C) MEG3 expression was inhibited by specific siRNAs and cell cycles distribution was examined by flow cytometry; (D) The bar chart represented the percentage of cells in $G_{0} / G_{1}, S$, and $\mathrm{G}_{2} / \mathrm{M}$ phase, as indicated. (E) 5-bromodeoxyuridine (BrdU) assay was performed to determine the proliferation of human PASMCs. MEG3 knockdown enhanced the BrdU incorporation compared with the siNC group both normal and hypoxic exposure; All experiments were performed in biological triplicates with three technical replicates. ${ }^{*} \mathrm{p}<$ $0.05,{ }^{* * *} \mathrm{p}<0.01$.

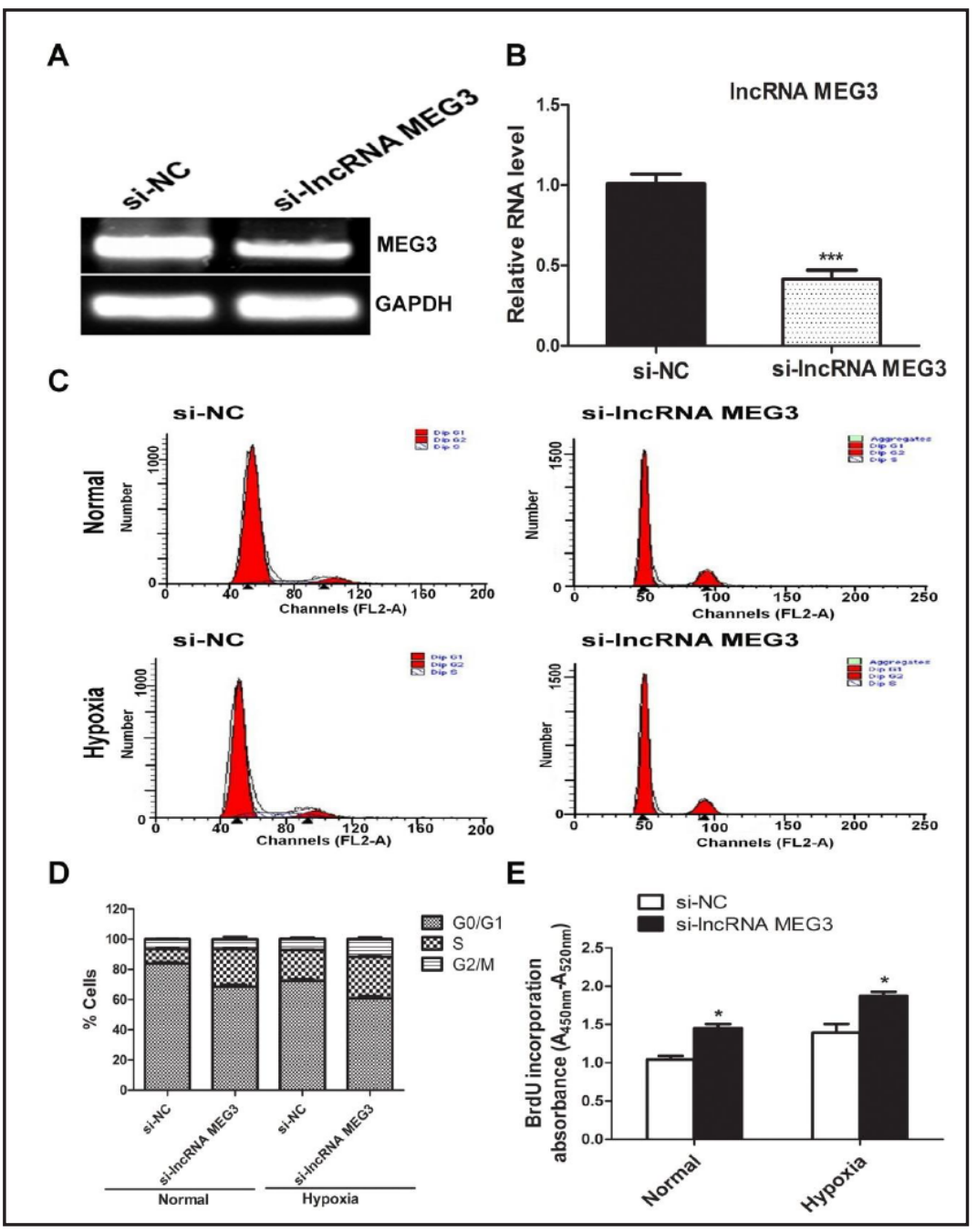

The efficiency of siRNA transfection and the inhibition of endogenous lncRNA MEG3 were confirmed by qRT-PCR (Fig. 2A, B). To investigate whether MEG3 regulates cell cycle progression, cell cycle was analyzed by flow cytometry. The results showed that MEG3 knockdown increased the percentage of cells in the $G_{2} / M+S$ phase both in normoxia and hypoxic conditions (Fig. 2C, D). To assess the population of cells, which are actively synthesizing DNA, BrdU incorporation assay was carried out. Our results showed higher fraction of proliferating cells in MEG3 knockdown cells compared with si-NC, and the difference is more obvious under hypoxic conditions (Fig. 2E). These results suggested that MEG3 plays a role in cell cycle regulation. Next, we examined the effects of MEG3 on cell migration capacity using wound healing assays. As shown in Fig. 3A, B, the migration capacity of si-lncRNA MEG3 cells were increased when compared to the si-NC cells, and similar results were observed under hypoxia condition. Taken together, these data indicate that hypoxia-induced MEG3 down-regulation may enhance the migration capacity of human PASMCs.

MEG3 knockdown induces PCNA, Cyclin A and Cyclin E protein expression

Cell cycle proteins play an important role in the both $\mathrm{S}$ and $\mathrm{G} 2 / \mathrm{M}$ phases, and have been generally regarded as a hallmark for cellular proliferation [22, 23]. To understand the molecular mechanism used by MEG3 to regulate cell cycle, western blotting was carried out to examine the expression level of cell cycle-associated proteins PCNA, Cyclin A, and Cyclin E. The results revealed that the expression of PCNA was increased by si-IncRNA MEG3 compared 
Fig. 3. MEG3 knockdown induces migration of human PASMCs. (A) Wound healing assays of human PASMCs after treatment with si-NC or si-lncRNA MEG3 both in normoxia and hypoxic conditions; (B) The histogram represents the migration distance for $24 \mathrm{~h}$. Results are representative of three independent experiments. ${ }^{*} \mathrm{p}<$ 0.05 .

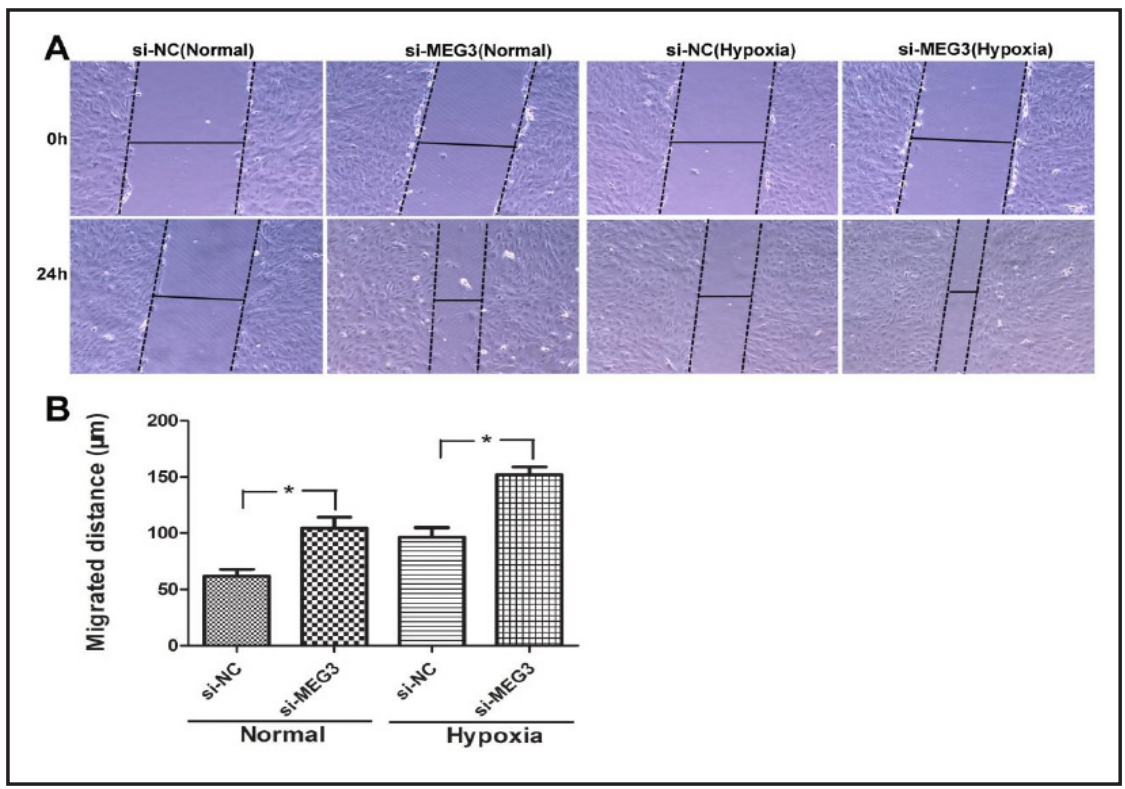

Fig. 4. MEG3 knockdown increases human PASMCs proliferation. $(A, C)$ Protein levels of PCNA, Cyclin A and Cyclin E were analysed by western blotting after transfection with si-lncRNA MEG3; (B, D, E) Densitometric quantification of protein bands showed a significant increase of PCNA, Cyclin A and Cyclin E after 24 hours transfection with MEG3 siRNA, whereas mismatch siRNA with only one nucleotide exchange (si-NC) showed no significant effect. Results are representative of three independent experiments. ${ }^{*} \mathrm{p}<0.05$.

A

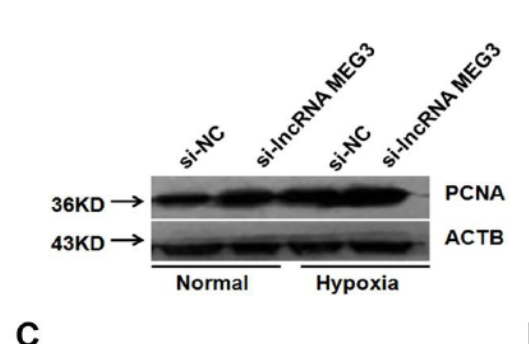

C

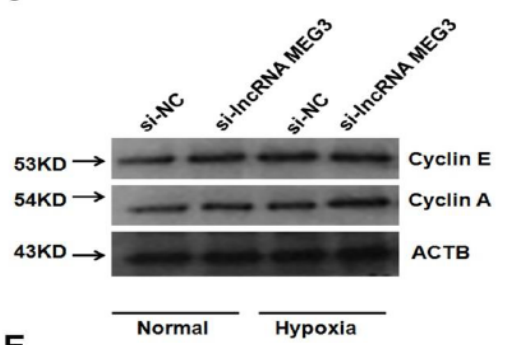

E

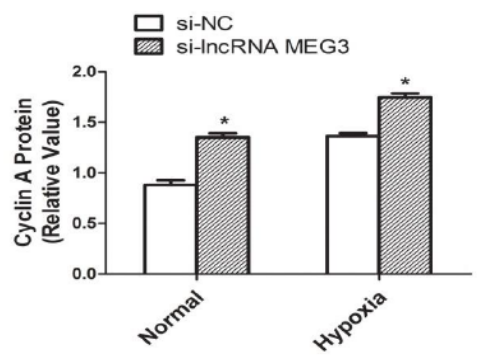

B

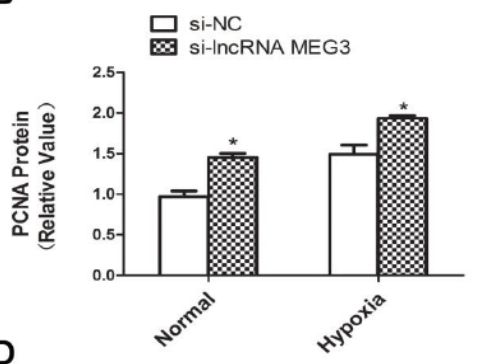

D

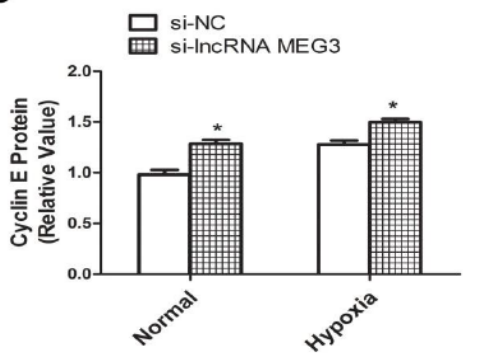

to si-NC in human PASMCs both in normal and hypoxic condition (Fig. 4A, B). We further examined whether MEG3 changed the expression of cell cycle regulators, including Cyclin A and Cyclin E, in human PASMCs after transfection of si-lncRNA MEG3. Western blotting displayed that expression of Cyclin A and Cyclin E was elevated by MEG3 knockdown, and results were consistent in hypoxia condition (Fig. 4C, D). 
Fig. 5. MEG3 stimulates activation of p53 protein. (A) Western blotting analysis of p53 protein level after si-lncRNA MEG3 or si-NC transfection; (B) Densitometric quantification of protein bands showed a significant decrease of p53 after 24 hours transfection with MEG3 siRNA, whereas mismatch siRNA with only one nucleotide exchange (siNC) showed no significant effect. (C) Immunofluorescence assay of p53 translocation during si-lncRNA MEG3 transfection. Red colour denotes p53 by rhodamine, and blue colour denotes nucleus by DAPI. (D) The expression of MDM2 protein was deteced in human PASMCs transfected with si-lncRNA MEG3 or si-NC; (E) Bar charts analysed the expression of MDM2. Results are representative of three independent experiments. ${ }^{*} \mathrm{p}<0.05$.

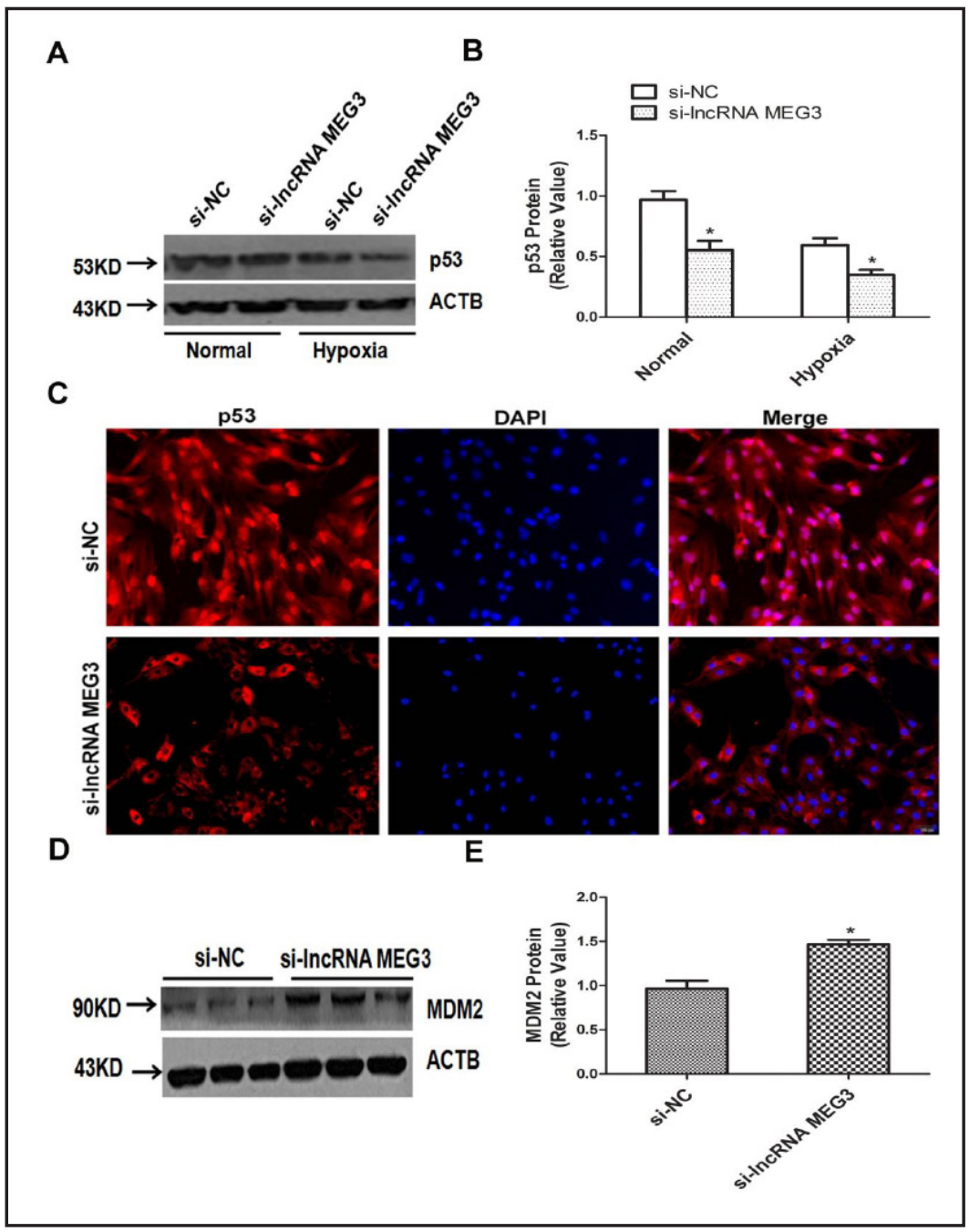

MEG3 stimulates activation of p53 protein

To further explore the mechanisms involved in MEG3 knockout induced cell proliferation, p53 western blotting and immunofluorescence were carried out after si-lncRNA MEG3 or siNC transfection into PASMCs. Recent studies have found that lncRNAs may play a significant role in the regulation of cell growth by modulating p53 pathway [19]. The results showed that the p53 expression was significantly decreased (Fig. 5A, B) and the expression of MDM2 was increased in human PASMCs transfected with si-lncRNA MEG3 compared to those with si-NC (Fig. 5D, E). P53 localizes to the nucleus, yet can be chaperoned to the cytoplasm by the negative regulator, mouse double minute 2 homolog (MDM2). MDM2 is an E3 ubiquitin ligase that is upregulated in the presence of active p53, where it poly-ubiquitinates p53 for proteasome targeting. We then performed immunofluorescence assay to detect p53 translocation. As shown in Fig. 5C, p53 was in nucleus under inactive state. In the presence of knockdown of MEG3 by siRNA-lncRNA MEG3, p53 was primarily located in the cytoplasm. These data confirm that MEG3 functions as apoptosis suppressor gene by regulating p53 activation in human PASMCs.

P53 pathway mediated the human PASMCs proliferation induced by MEG3

Previous studies have suggested that MEG3 increased the p53 activity in cancer cells [24, 25]. Because pulmonary hypertension and cancers have similar pathological processes, like cell proliferation increased and apoptosis decreased. We speculate that the MEG3 may induce 
Fig. 6. MEG3 regulates human PASMC proliferation by p53 pathway. (A) CCK-8 assays showed that si-lncRNA MEG3 increased human PASMCs proliferation. However, treatment with p53 overexpression markedly inhibited cell proliferation; (B) BrdU assays showed that si-lncRNA MEG3 increased human PASMCs proliferation. Overexpression of p53 decreased the BrdU incorporation compared with the lncRNA-MEG3 siRNA group; (C, D) Upon the transfection of NC siRNA, MEG3 siRNA or MEG3 siRNA+p53, the percentage of cells in G0/ G1, S and G2/M phase of cell cycle was quantified by flow cytometer analysis; (E) The increase of PCNA expression induced by IncRNA MEG3 siRNA was suppressed after treating the cells with overexpressed p53; (F) LncRNA MEG3 knockdown increased the expression of Cyclin A and CyclinE, and the expression of Cyclin A and CyclinE was decreased after overexpression of p53. Results are representative of three independent experiments. ${ }^{*} \mathrm{p}<$ 0.05 .
A
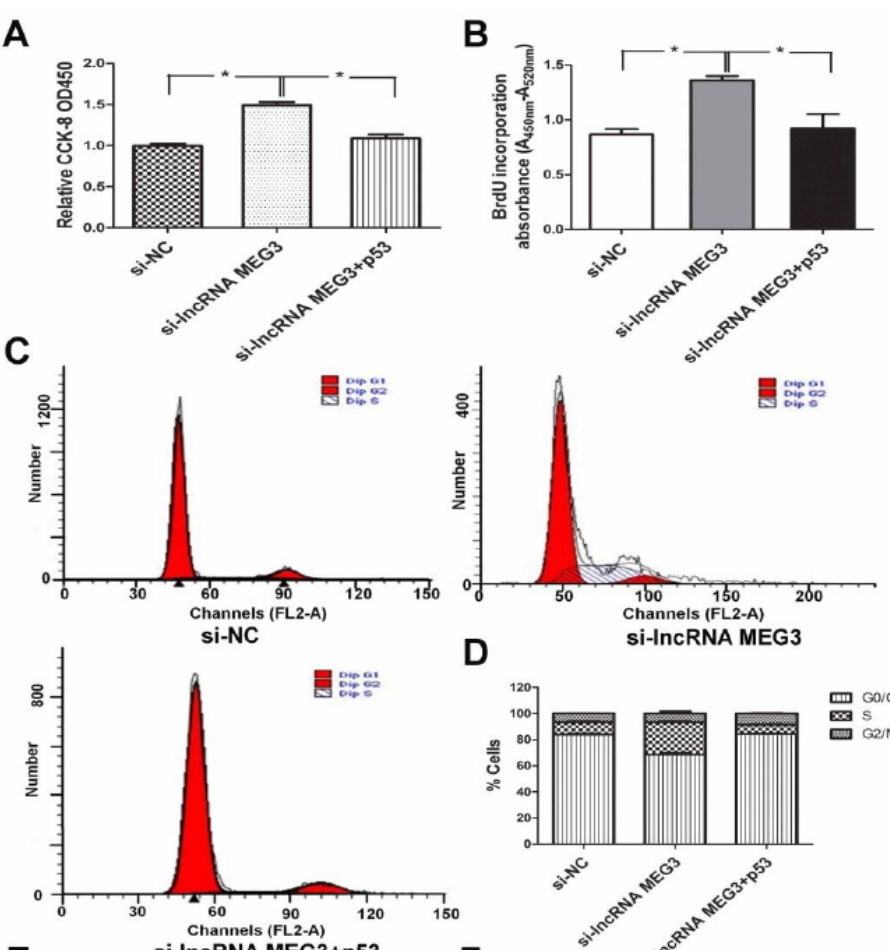

E
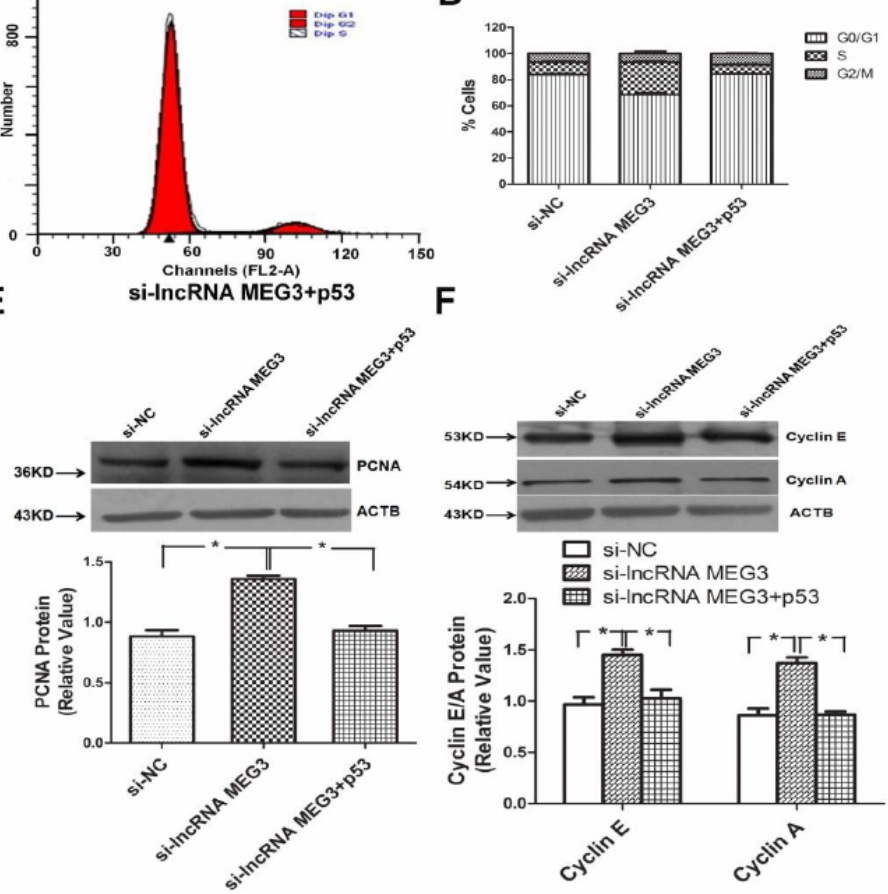

human PASMCs proliferation by activation of the p53 pathway. To investigate the interplay of p53 and IncRNA-MEG3 on cell proliferation, we overexpressed p53 in human PASMCs.CCK-8 assays showed that si-lncRNA MEG3 significantly enhanced the growth of human PASMCs compared to si-NC. In contrast, si-lncRNA MEG3 with p53 overexpression statistically reduced cell number human PASMCs compared with si-lncRNA MEG3 (Fig. 6A). Figure 6B demonstrated that the increased effect of si-IncRNA MEG3 on cell BrdU incorporation was significantly suppressed by overexpression of p53. To elucidate whether the p53 pathway participates in MEG3-mediated cell cycle activity, the number of cells in the different cell cycle phases was detected by flow cytometry. As shown in Fig. 6C, D, the amount of G2/M + S phase cells increased under MEG3 knockdown, p53 overexpression suppressed the cell cycle progression and induced a cell cycle arrest at the G0/G1phase in PASMCs. We also analyzed the expression of the PCNA in human PASMCs. We found that MEG3 knockdown increased the expression of PCNA, and the effect was decreased in the presence of p53 (Fig. 6E). To understand the role of p53 in cell cycle progression, the expression of Cyclin A and Cyclin E were studied in human PASMCs. The results show that si-lncRNA MEG3 increased the 


\section{Cellular Physiology Cell Physiol Biochem 2017;42:2569-2581

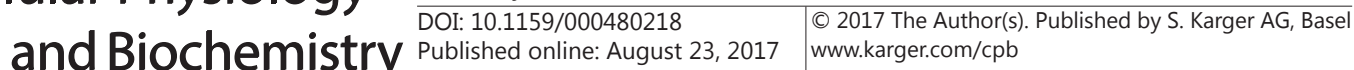

Sun et al.: Role of MEG3 in Pulmonary Arterial Hypertension

expression of cyclin A and cyclin E and that the effects were significantly decreased after p53 overexpression (Fig. 6E). Together, these results demonstrate that lncRNA-MEG3 functions through the p53 pathway to regulate cell proliferation.

\section{Discussion}

Pulmonary arterial hypertension is characterized by a progressive increase in pulmonary vascular resistance and obliterative pulmonary vascular remodeling which mechanism is complicated and still unknown. Our studies discovered that lncRNA MEG3 was low-expressed in PAH patients and human PASMCs in hypoxia. MEG3 regulates PASMCs function in vitro. Moreover, we found that MEG3 could activate the p53 signaling pathways, and its intervention affected p53 nuclear translocation. Thus, lncRNA MEG3 is a promising therapeutic strategy for treating PAH and vascular remodeling.

LncRNAs, which were defined as transcripts $>200$ nucleotides in length, were once considered to be a by-product of RNA polymerase II transcription and transcriptional "noise" without biological function. Accompanied by the research progress of IncRNAs, a growing body of study has revealed that IncRNAs play a center control role in a wide range of biological processes, including development, differentiation, proliferation, apoptosis, and so on [26-28]. Disregulation of lncRNAs is associated with several human diseases, including cardiovascular diseases [29]. For example, braveheart was found as critical regulator of cardiovascular commitment from nascent mesoderm, which represented the first example of IncRNA with potential implications in cardiovascular development [8]. LncRNA (cardiac hypertrophy related factor, CHRF) regulates cardiac hypertrophy by targeting miR-489 [30]. In vascular smooth muscle cells (VSMC), lincRNA-p21 and IncRNA SENCR regulate VSMCs proliferation, apoptosis, migration, and phenotypic switching [19, 31]. Pulmonary arterial hypertension is usually characterized by abnormal pulmonary vascular remodeling, which is regulated by vascular smooth muscle-mediated proliferation, migration and major phenotypic changes from the contractile to the synthetic subtype [32]. We speculated that lncRNAs might regulate pulmonary vascular remodeling via affecting PASMCs proliferation and migration. MEG3 is located at chromosome 14q32.3 in human genome, which is an imprinted gene belonging to the imprinted DLK1-MEG3 locus. MEG3 was identified as the human counterpart of a mouse imprinted gene Gtl2 [33]. Mice died in perinatally and had major skeletal muscle defects when maternal Meg3 region was deleted. MEG3 is expressed in many normal tissues, and the loss of MEG3 expression has been observed in various types of cancers, including non-small cell lung cancer (NSCLC) [10], gastric cancer [11] and bladder cancer [12]. In addition, IncRNA MEG3 can regulate endothelial cell function by hypoxia [34]. At present, the importance in cell biology and role in the development of the disease of MEG3 have been in continuous study. However, the involvement of MEG3 in PAH has not been reported. We found that MEG3 knockdown affects human PASMCs proliferation and migration, and upregulates the proportion of cells in the $S$ and $G_{2} / M$ phases. The process may be through direct or indirect stimulation of PCNA and Cyclins. All of the information presented here suggested that MEG3 lower expression activates cell cycle progression in the process of PASMCs proliferation. Thus, we speculated that MEG3-mediated PASMCs function is involved in the pathogenesis of vascular remodeling in PAH.

It is well-known that 553 plays an important role on the pathogenesis of $\mathrm{PAH}[35,36]$. The expression and transcriptional activity of p53 is tightly regulated at multiple levels. In particular, post-transcriptional regulation is necessary for the function of $\mathrm{p} 53$ proteins by ubiquitination and acetylation. MDM2, a direct transcriptional target gene of p53, seems to participate in both pathways. MDM2 is a pivotal regulator of the fate and activity of p53, then p53 is controlled through regulation of protein expression level via MDM2, establishing a p53/MDM2 negative feedback loop [37, 38]. To investigate the mechanism of p53 activation by MEG3, the expression level of p53 was measured using western blotting analysis. Interestingly, MEG3 knockdown significantly decreased the expression of p53 protein 
in PASMCs both normoxia and hypoxic condition. P53 localizes to the nucleus, yet can be chaperoned to the cytoplasm in active state. We performed immunofluorescence assay to detect p53 translocation. In cultured PASMCs, we have found that p53 is in nucleus under siNC group. In the presence of knockdown of MEG3 by siRNA-MEG3, p53 was primarily located in the cytoplasm. In addition, our results showed that MDM2, a negative regulator of $\mathrm{p} 53$, was increased in siRNA-MEG3 group. Thus, our data provide a new molecular mechanism that MEG3 regulates PASMCs function and pulmonary vascular remodeling through p53 pathway.

Signaling by p53 directs cell proliferation, cell polarity, cell cycle and apoptosis control, also plays a central role in PAH $[35,36]$. Our experiments also demonstrate that MEG3 knockdowm induced PASMCs proliferation is directly regulated by p53 pathway. We observed that si-lncRNA MEG3 was a stimulus for increasing activation of p53 in PASMCs and resultant cell proliferation. The critical role of p53 in MEG3-induced PASMCs proliferation was confirmed by p53 overexpression. Assessment of the cell cycle distribution showed that MEG3 knockdowm reduced the percentage of cells in $G_{0} / G_{1}$ phase and increased the number of cells in $G_{2} / M$ and $S$ phases. Si-lncRNA MEG3+p53 group opposed the effect of si-lncRNA MEG3 by increasing the percentage of cells in $G_{0} / G_{1}$ phase and decreasing the percentage of cells in $\mathrm{G}_{2} / \mathrm{M}$ and $\mathrm{S}$ phases. In addition, overexpression of p53 inhibits the expression of PCNA, Cyclin A and Cyclin E. These results thus provide a solid evidence for a role of p53dependent mechanism and activation in the mitogenic effect of MEG3. Overall, these data suggest that lncRNA MEG3 may contribute to PASMCs proliferation via the activation of p53 signaling pathway.

In conclusion, our findings indicate that MEG3 plays a key role in the pulmonary vascular remodeling in PAH. MEG3 regulates PASMCs function through p53 signaling. Because vascular remodeling is now recognized as a major prognostic marker in patients at $\mathrm{PAH}, \mathrm{MEG} 3$ may represent an interesting pharmacological target for the treatment of PAH conditions.

\section{Abbreviations}

LncRNAs (long non-coding RNAs); PAH (pulmonary arterial hypertension); PASMCs (pulmonary artery smooth muscle cells); MEG3 (maternally expressed gene 3).

\section{Acknowledgments}

The study is supported by funds from the Natural Science Foundation of Jiangsu Province (BK20161297), project of Jiangsu Provincial Commission of Health and Family Planning (QNRC2016505), the Social Fund for Development of Lianyungang Science and Technology Bureau (SH1612), the Dr. Start-up Fund of Lianyungang First People's Hospital (BS15012).

\section{Disclosure Statement}

The authors declare no conflict of interest.

\section{References}

1 Tuder RM: Pathology of pulmonary arterial hypertension. Semin Respir Crit Care Med 2009;30:376-385.

2 Rabinovitch M: Molecular pathogenesis of pulmonary arterial hypertension. J Clin Invest 2008;118:23722379. 


\section{Cellular Physiology Cell Physiol Biochem 2017;42:2569-2581 \begin{tabular}{l|l|l} 
and Biochemistry Published online: August 23, 2017 & $\begin{array}{l}\text { (c) } 2017 \text { The Author(s). Published by S. Karger AG, Basel } \\
\text { www.karger.com/cpb }\end{array}$
\end{tabular}}

3 Zhang E, Han L, Yin D, He X, Hong L, Si X, Qiu M, Xu T, De W, Xu L, Shu Y, Chen J: H3K27 acetylation activated-long non-coding RNA CCAT1 affects cell proliferation and migration by regulating SPRY4 and HOXB13 expression in esophageal squamous cell carcinoma. Nucleic Acids Res 2017;45:3086-3101.

-4 Guttman M, Donaghey J, Carey BW, Garber M, Grenier JK, Munson G, Young G, Lucas AB, Ach R, Bruhn L, Yang X, Amit I, Meissner A, Regev A, Rinn JL, Root DE, Lander ES: lincRNAs act in the circuitry controlling pluripotency and differentiation. Nature 2011;477:295-300.

5 Ginger MR, Shore AN, Contreras A, Rijnkels M, Miller J, Gonzalez-Rimbau MF, Rosen JM: A noncoding RNA is a potential marker of cell fate during mammary gland development. Proc Natl Acad Sci USA 2006;103:5781-5786.

-6 Khaitan D, Dinger ME, Mazar J, Crawford J, Smith MA, Mattick JS, Perera RJ: The melanoma-upregulated long noncoding RNA SPRY4-IT1 modulates apoptosis and invasion. Cancer Res 2011;71:3852-3862.

7 Kataoka M, Wang DZ: Non-Coding RNAs Including miRNAs and lncRNAs in Cardiovascular Biology and Disease. Cells 2014;3:883-898.

8 Klattenhoff CA, Scheuermann JC, Surface LE, Bradley RK, Fields PA, Steinhauser ML, Ding H, Butty VL, Torrey L, Haas S, Abo R, Tabebordbar M, Lee RT, Burge CB, Boyer LA: Braveheart, a long noncoding RNA required for cardiovascular lineage commitment. Cell 2013;152:570-583.

9 Benetatos L, Vartholomatos G, Hatzimichael E: MEG3 imprinted gene contribution in tumorigenesis. Int J Cancer 2011;129:773-779.

10 Lu KH, Li W, Liu XH, Sun M, Zhang ML, Wu WQ Xie WP, Hou YY: Long non-coding RNA MEG3 inhibits NSCLC cells proliferation and induces apoptosis by affecting p53 expression. BMC Cancer 2013;13:461.

11 Sun M, Xia R, Jin F, Xu T, Liu Z, De W, Liu X: Downregulated long noncoding RNA MEG3 is associated with poor prognosis and promotes cell proliferation in gastric cancer. Tumour Biol 2014;35:1065-1073.

12 Ying L, Huang Y, Chen H, Wang Y, Xia L, Chen Y, Liu Y, Qiu F: Downregulated MEG3 activates autophagy and increases cell proliferation in bladder cancer. Mol Biosyst 2013;9:407-411.

13 Peng W, Si S, Zhang Q Li C, Zhao F, Wang F, Yu J, Ma R: Long non-coding RNA MEG3 functions as a competing endogenous RNA to regulate gastric cancer progression. J Exp Clin Cancer Res 2015;34:79.

14 Luo G, Wang M, Wu X, Tao D, Xiao X, Wang L, Min F, Zeng F, Jiang G: Long Non-Coding RNA MEG3 Inhibits Cell Proliferation and Induces Apoptosis in Prostate Cancer. Cell Physiol Biochem 2015;37:2209-2220.

15 Zhou Y, Zhong Y, Wang Y, Zhang X, Batista DL, Gejman R, Ansell PJ, Zhao J, Weng C, Klibanski A: Activation of p53 by MEG3 non-coding RNA. J Biol Chem 2007;282:24731-24742.

16 Voelkel NF, Cool C, Lee SD, Wright L, Geraci MW, Tuder RM: Primary pulmonary hypertension between inflammation and cancer. Chest 1998;114:225s-230s.

17 Archer SL, Weir EK, Wilkins MR: Basic science of pulmonary arterial hypertension for clinicians: new concepts and experimental therapies. Circulation 2010;121:2045-2066.

18 Liu Y, Cao Y, Sun S, Zhu J, Gao S, Pang J, Zhu D, Sun Z: Transforming growth factor-beta1 upregulation triggers pulmonary artery smooth muscle cell proliferation and apoptosis imbalance in rats with hypoxic pulmonary hypertension via the PTEN/AKT pathways. Int J Biochem Cell Biol 2016;77:141-154.

19 Wu G, Cai J, Han Y, Chen J, Huang ZP, Chen C, Cai Y, Huang H, Yang Y, Liu Y, Xu Z, He D, Zhang X, Hu X, Pinello L, Zhong D, He F, Yuan GC, Wang DZ, Zeng C: LincRNA-p21 regulates neointima formation, vascular smooth muscle cell proliferation, apoptosis, and atherosclerosis by enhancing p53 activity. Circulation 2014;130:1452-1465.

20 Livak KJ, Schmittgen TD: Analysis of relative gene expression data using real-time quantitative PCR and the 2(-Delta Delta C(T)) Method. Methods 2001;25:402-408.

21 Yu X, Li T, Liu X, Yu H, Hao Z, Chen Y, Zhang C, Liu Y, Li Q Mao M, Zhu D: Modulation of Pulmonary Vascular Remodeling in Hypoxia: Role of 15-LOX-2/15-HETE-MAPKs Pathway. Cell Physiol Biochem 2015;35:20792097.

22 Liu Y, Ma C, Zhang Q, Yu L, Ma J, Zhang L, Hao X, Cao F, Wang L, Zhu D: The key role of transforming growth factor-beta receptor I and 15-lipoxygenase in hypoxia-induced proliferation of pulmonary artery smooth muscle cells. Int J Biochem Cell Biol 2012;44:1184-1202.

23 Ma C, Li Y, Ma J, Liu Y, Li Q, Niu S, Shen Z, Zhang L, Pan Z, Zhu D: Key role of 15-lipoxygenase/15hydroxyeicosatetraenoic acid in pulmonary vascular remodeling and vascular angiogenesis associated with hypoxic pulmonary hypertension. Hypertension 2011;58:679-688.

24 Zhang J, Yao T, Wang Y, Yu J, Liu Y, Lin Z: Long noncoding RNA MEG3 is downregulated in cervical cancer and affects cell proliferation and apoptosis by regulating miR-21. Cancer Biol Ther 2016;17:104-113. 


\section{Cellular Physiology Cell Physiol Biochem 2017;42:2569-2581

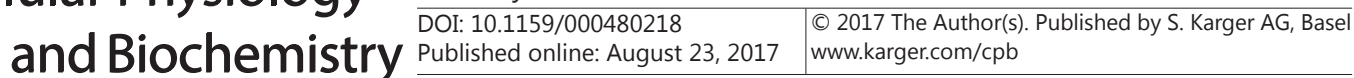

-25 Chen RP, Huang ZL, Liu LX, Xiang MQ, Li GP, Feng JL, Liu B, Wu LF: Involvement of endoplasmic reticulum stress and p53 in lncRNA MEG3-induced human hepatoma HepG2 cell apoptosis. Oncol Rep 2016;36:16491657.

26 Tripathi V, Ellis JD, Shen Z, Song DY, Pan Q, Watt AT, Freier SM, Bennett CF, Sharma A, Bubulya PA, Blencowe BJ, Prasanth SG, Prasanth KV: The nuclear-retained noncoding RNA MALAT1 regulates alternative splicing by modulating SR splicing factor phosphorylation. Mol Cell 2010;39:925-938.

-27 Wang KC, Yang YW, Liu B, Sanyal A, Corces-Zimmerman R, Chen Y, Lajoie BR, Protacio A, Flynn RA, Gupta RA, Wysocka J, Lei M, Dekker J, Helms JA, Chang HY: A long noncoding RNA maintains active chromatin to coordinate homeotic gene expression. Nature 2011;472:120-124. Harries LW: Long non-coding RNAs and human disease. Biochem Soc Trans 2012;40:902-906.

-29 Greco S, Gorospe M, Martelli F: Noncoding RNA in age-related cardiovascular diseases. J Mol Cell Cardiol 2015;83:142-155.

-30 Wang K, Liu F, Zhou LY, Long B, Yuan SM, Wang Y, Liu CY, Sun T, Zhang XJ, Li PF: The long noncoding RNA CHRF regulates cardiac hypertrophy by targeting miR-489. Circ Res 2014;114:1377-1388.

31 Thum T, Kumarswamy R: The smooth long noncoding RNA SENCR. Arterioscler Thromb Vasc Biol 2014;34:1124-1125.

-32 Tuder RM: Pulmonary vascular remodeling in pulmonary hypertension. Cell Tissue Res 2017;367:643-649.

33 Zhang X, Gejman R, Mahta A, Zhong Y, Rice KA, Zhou Y, Cheunsuchon P, Louis DN, Klibanski A: Maternally expressed gene 3, an imprinted noncoding RNA gene, is associated with meningioma pathogenesis and progression. Cancer Res 2010;70:2350-2358.

-34 Michalik KM, You X, Manavski Y, Doddaballapur A, Zornig M, Braun T, John D, Ponomareva Y, Chen W, Uchida S, Boon RA, Dimmeler S: Long noncoding RNA MALAT1 regulates endothelial cell function and vessel growth. Circ Res 2014;114:1389-1397.

-35 Mizuno S, Bogaard HJ, Kraskauskas D, Alhussaini A, Gomez-Arroyo J, Voelkel NF, Ishizaki T: p53 Gene deficiency promotes hypoxia-induced pulmonary hypertension and vascular remodeling in mice. Am J Physiol Lung Cell Mol Physiol 2011;300:L753-761.

-36 Mouraret N, Marcos E, Abid S, Gary-Bobo G, Saker M, Houssaini A, Dubois-Rande JL, Boyer L, Boczkowski J, Derumeaux G, Amsellem V, Adnot S: Activation of lung p53 by Nutlin-3a prevents and reverses experimental pulmonary hypertension. Circulation 2013;127:1664-1676.

37 Momand J, Zambetti GP, Olson DC, George D, Levine AJ: The mdm-2 oncogene product forms a complex with the p53 protein and inhibits p53-mediated transactivation. Cell 1992;69:1237-1245.

-38 Chen J, Marechal V, Levine AJ: Mapping of the p53 and mdm-2 interaction domains. Mol Cell Biol 1993;13:4107-4114. 